\title{
Long non-coding RNA BLACAT1 promotes the proliferation and invasion of glioma cells via Wnt/ $\beta$-catenin signaling
}

\author{
XIUSHAN LI ${ }^{1}$, SHUJING QI ${ }^{2}$, DONGZHOU MA ${ }^{1}$, JINBIAO FAN ${ }^{1}$ and JINGTAO WANG ${ }^{1}$ \\ Departments of ${ }^{1}$ Neurosurgery and ${ }^{2}$ Nutrition, Affiliated Hospital of Hebei University of Engineering, Handan, \\ Hebei 056002, P.R. China
}

Received July 24, 2018; Accepted March 7, 2019

DOI: $10.3892 /$ etm.2019.7468

\begin{abstract}
Long non-coding RNAs (lncRNAs) are hypothesized to regulate numerous biological behaviors in human cancers. The present study aimed to explore the roles of lncRNA bladder cancer associated transcript 1 (BLACAT1) in glioma. The expression of BLACAT1 in glioma tissues and cell lines was determined by reverse transcription-quantitative polymerase chain reaction (RT-qPCR). CCK-8 assay, colony formation assay, wound healing assay and Transwell invasion assay were used to explore the roles of BLACAT1 in glioma cells. RT-qPCR and western blot analysis were used to determine the BLACAT1 molecular mechanism. The findings demonstrated that IncRNA BLACAT1 was overexpressed in glioma tissues and cell lines. High BLACAT1 expression was correlated with high tumor grade in glioma patients. Functional assays determined that BLACAT1 promoted glioma cell proliferation, migration, invasion and epithelial-mesenchymal transition in vitro. In addition, it was demonstrated that BLACAT1 activated the $\mathrm{Wnt} / \beta$-catenin signaling pathway. In conclusion, BLACAT1 may serve as an oncogenic lncRNA in glioma progression via activation of the Wnt/ $\beta$-catenin signaling pathway. Therefore, BLACAT1 may be a novel therapeutic target for glioma treatment.
\end{abstract}

\section{Introduction}

Glioma is the most common malignant tumor of the central nervous system with a high recurrence rate and low survival rate $(1,2)$. Despite the great advances in treatment strategies over the last decades, the prognosis of glioma remains unsatisfactory $(3,4)$. Therefore, a deeper understanding of the

Correspondence to: Dr Jingtao Wang, Department of Neurosurgery, Affiliated Hospital of Hebei University of Engineering, 81 Congtai Road, Handan, Hebei 056002, P.R. China E-mail: jingtaowangwjt@163.com

Key words: glioma, long non-coding RNA, bladder cancer associated transcript 1, proliferation, invasion, epithelial-mesenchymal transition, Wnt $/ \beta$-catenin molecular mechanisms involved in glioma tumorigenesis is urgently required.

Genome-wide sequencing has revealed that up to $98 \%$ of the genome are non-coding genes with $<2 \%$ of genes encoding proteins $(5,6)$. Among these non-transcribed genes, long non-coding RNAs (lncRNAs), which consist of $>200$ nucleotides, have been demonstrated to have critical roles in various cellular processes $(7,8)$. Mechanistically, lncRNAs regulate gene expression via multiple mechanisms including gene imprinting, epigenetic modification and microRNA (miRNA) degradation (9-11). Recent studies have characterized a number of lncRNAs in the initiation of glioma. For example, Feng et al (12) demonstrated that upregulation of the lncRNA, RNA component of mitochondrial RNA processing endoribonuclease, could promote glioma cell proliferation and invasion. Wang et al (13) demonstrated that the IncRNA, Prader Willi/Angelman region RNA 5, inhibits proliferation and progression of glioma through interaction with enhancer of zeste 2 polycomb repressive complex 2 subunit. Ma et al (14) determined that the IncRNA, differentiation antagonizing non-protein coding RNA, mediates cisplatin resistance in glioma cells via activating the AXL receptor tyrosine kinase/phosphoinositide 3-kinase/protein kinase $\mathrm{B} /$ nucleur factor- $\mathrm{\kappa B}$ signaling pathway. However, the roles and underlying mechanisms through which lncRNAs modulate glioma progression remain largely unknown.

The present study explored BLACAT1 expression both in glioma tissues and cell lines. In vitro functional assays were used to explore the roles of BLACAT1 on glioma progression. Western blot analysis and reverse transcription-quantitative polymerase chain reaction (RT-qPCR) were used to determine the underlying mechanism of BLACAT1 on glioma cell progression.

\section{Patients and methods}

Tissue samples. A total of 35 paired glioma tissues and adjacent non-tumor tissues (16 female and 19 male patients; mean age, $49.61 \pm 15.51$ years) were obtained from patients with glioma between August 2016 and December 2017 at the Department of Neurosurgery, Affiliated Hospital of Hebei University of Engineering (Handan, China). None of the patients received preoperative treatment. Tissues were immediately frozen in liquid nitrogen following the surgical resection and stored at 
$-80^{\circ} \mathrm{C}$. Informed consent was obtained from each patient. This study was approved by the Ethics Committee of Affiliated Hospital of Hebei University of Engineering.

Cell culture and transfection. The normal human astrocyte (NHA) cells and glioma cell lines (U251, T98G, H4, A172 and LN229) were obtained from the American Type Culture Collection (Manassas, VA, USA). Cells were cultured in Dulbecco's modified Eagle's medium (DMEM) containing $10 \%$ fetal bovine serum (FBS; Thermo Fisher Scientific, Inc., Waltham, MA, USA), and incubated under standard conditions $\left(37^{\circ} \mathrm{C}, 5 \% \mathrm{CO}_{2}\right)$.

The small interfering RNAs (siRNAs) targeting BLACAT1 (si-BLACAT1) and scrambled negative control siRNA (si-NC) were synthesized by Genepharma (Shanghai, China). The si-BLACAT1 sequences were as follows: si-BLACAT1-1 5'-AGGCUGGUUUCUGCCCUCAUCCUU U-3'; si-BLACAT1-2, 5'-GCCCAGCUUCUAGUCCUCUCC UUAU-3'; and si-NC, 5'-AATTCTCCGAACGTGTCACGT-3'. Cell transfection was performed by using Lipofectamine 2000 (Thermo Fisher Scientific, Inc.) according to the manufacturer's introductions. The transfection was performed $48 \mathrm{~h}$ prior to the subsequent analysis.

Reverse transcription-quantitative polymerase chain reaction. Total RNA was extracted from tissues or cells using TRIzol reagent (Invitrogen) according to the manufacturer's instructions. Then, the reverse transcription was done with a Reverse Transcription kit (Takara Bio, Inc., Otsu, Japan). qPCR analysis was performed with SYBR Green (Takara Bio, Inc.). The relative expression levels were calculated by the $2^{-\triangle \Delta C q}$ method (15) and normalized to GAPDH. qPCR reactions were performed using the ABI7500 system (Applied Biosystems; Thermo Fisher Scientific, Inc., Waltham, MA, USA). The thermocycling conditions of qPCR were as follows: $94^{\circ} \mathrm{C}$ for $15 \mathrm{~min}$, followed by 45 cycles at $94^{\circ} \mathrm{C}$ for $10 \mathrm{sec}, 60^{\circ} \mathrm{C}$ for $30 \mathrm{sec}$ and $72^{\circ} \mathrm{C}$ for $30 \mathrm{sec}$. The primer sequences were as follows: Vimentin, $5^{\prime}$-AATTTCACG CAGAGCAACAG-3' and 5'-CCACTAAGGCAGCACGTA AA-3'; E-cadherin, 5'-TTAACCTCACCAATCCTTGCT-3' and 5'-TAGCCCATTTCTTCCCAATC-3'; and N-cadherin, 5'-AACCTAGCCTACTGGCCAAA-3' and 5'-AACATC GAGGTCGTAAACCC-3'.

Cell proliferation assays. Cell proliferation was measured by CCK-8 (Roche Diagnostics, Basel, Switzerland) and colony formation assay. For CCK- 8 assay, cells $\left(1 \times 10^{3} /\right.$ well) were plated in the 96-well plates (Corning Inc., Corning, NY, USA) then $10 \mu \mathrm{l}$ of CCK-8 was added to each well at the time of harvest. After $2 \mathrm{~h}$, the absorbance was recorded at $450 \mathrm{~nm}$ to determine cell viability. For the colony formation assay, cells were seeded in the 6-well plate at 1,000 cells per well and cultured for 10 days. Colonies were fixed with methanol and stained with $0.1 \%$ crystal violet. The colonies were observed and counted with microscope (magnification, x40; Nikon Corporation, Tokyo, Japan).

Cell apoptosis analysis. Cells were harvested following transfection were harvested and stained with propidium iodide (PI) for $30 \mathrm{~min}$. The fluorescein isothiocyanate (FITC)-Annexin V
Apoptosis Detection kit (BD Biosciences, San Jose, CA, USA) based on double staining with FITC-Annexin V and PI was applied to detect the apoptosis level. Then apoptotic cells were analyzed with a FC500 flow cytometer equipped with Cell Quest 3.0 software (BD Biosciences, Franklin Lakes, NY, USA).

Wound-healing assay. Cells were seeded in 6-well plates, cultured to $\sim 90 \%$ confluence, then a wound was generated by scraping the cells with a $200 \mu \mathrm{l}$ tip. The cells were washed with PBS to remove debris and cultured with DMEM medium containing $10 \%$ FBS. After $24 \mathrm{~h}$, the wound healing of different groups was observed using microscopy. (magnification, x100; Nikon Corporation).

Transwell invasion assay. The Transwell invasion assay was performed using Matrigel-coated (BD Biosciences) 24-well Transwell chambers (Corning Inc.). Cells were resuspended in serum-free $200 \mu 1$ DMEM medium at a density of $5 \times 10^{4}$ cells/well into the upper chamber of Transwell cell culture chambers. The lower chambers of the Transwell were filled with DMEM supplemented with $10 \%$ FBS. Following incubation for $24 \mathrm{~h}$, cells on the upper surface of the filter were removed using a cotton swab. Cells migrating through the filter to the lower surface were fixed with $4 \%$ paraformaldehyde and stained with $0.1 \%$ crystal violet for $10 \mathrm{~min}$. The cells on the bottom of the membrane were calculated from five random light microscopic fields using a light microscope (magnification, x100; Nikon Corporation).

Western blot analysis. Protein lysates were prepared using radioimmunoprecipitation assay buffer (Beyotime Institute of Biotechnology, Shanghai, China) and the concentration was measured with a bicinchoninic acid protein assay kit (Beyotime Institute of Biotechnology). Then, $40 \mu \mathrm{g}$ (loaded per lane) protein samples were separated by electrophoresis in 10\% SDS-polyacrylamide gel and then transferred onto a polyvinylidene difluoride membrane. The membranes were incubated with primary antibody against $\mathrm{N}$-cadherin (cat. no. ab76011; 1:1,000), Vimentin (cat. no. ab71144; 1:1,000), E-cadherin (cat. no. ab133597; 1:1,000), $\beta$-catenin (cat. no. ab27798; 1:1,000), Cyclin D1 (cat. no. ab21699, 1:1,000) and GAPDH (cat. no. b37168, 1:5,000; all Abcam, Cambridge, UK) at $4^{\circ} \mathrm{C}$ overnight. The membrane was washed for three times with Tris-buffered saline and Polysorbate 20 and incubated with horseradish peroxidase-labeled secondary antibody goat anti-rabbit (cat. no. ab97051; 1:2,000 dilution; Abcam) for $1 \mathrm{~h}$ at room temperature. The bands were visualized using enhanced chemiluminescent reagent (Millipore, Bedford, MA, USA) and the bands were quantified by densitometry using ImageJ software (V1.8.0; National Institutes of Health, Bethesda, MD, USA).

Statistical analysis. All data were presented as mean \pm standard deviation. Statistical analysis was performed using the SPSS software package (SPSS, Inc., Chicago, IL, USA). Statistical significance was assessed by Student's t-test or one-way analysis of variance was applied followed by Least Significance Difference post hoc test. $\mathrm{P}<0.05$ was considered to indicate statistical significance. 
A

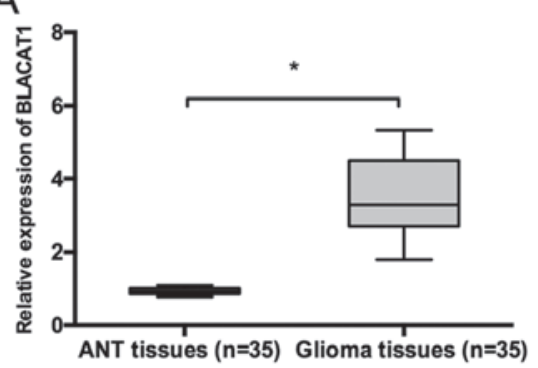

B

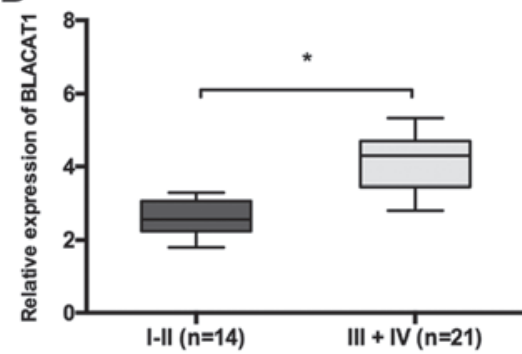

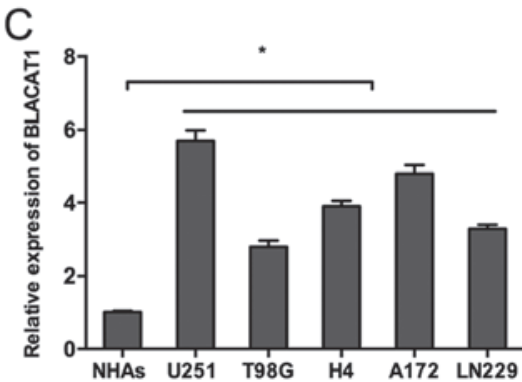

Figure 1. BLACAT1 is highly expressed in glioma. (A) RT-qPCR was used to determine the expression of BLACAT1 in glioma tissues and adjacent non-tumor tissues. (B) Expression of BLACAT1 in glioma patients with different tumor grades. (C) The expression of BLACAT1 was detected in glioma cell lines (U251, T98G, H4, A172 and LN229) and NHAs by qRT-PCR. "P<0.05 with comparisons indicated by lines. BLACAT1, bladder cancer associated transcript 1; RT-qPCR, reverse transcription-quantitative polymerase chain reaction; NHA, normal human astrocytes; ANT, adjacent non-tumor.

\section{Results}

LncRNA BLACAT1 is upregulated in glioma. BLACAT1 mRNA expression in glioma was determined by RT-qPCR. Results demonstrated that BLACAT1 mRNA expression was significantly increased in glioma tissues compared to adjacent non-tumor (ANT) tissues (Fig. 1A; P<0.05). BLACAT1 mRNA expression was also markedly increased in glioma tumors of high grade (III-IV) compared with low grade (I-II) tumors (Fig. 1B; $\mathrm{P}<0.05$ ). The upregulation of BLACAT1 in glioma was further verified in glioma cell lines by RT-qPCR. Results determined that BLACAT1 was highly expressed in glioma cell lines (U251, T98G, H4, A172 and LN229) compared with the NHA cell line. U251 and A172 cells were selected for further assays due to displaying the highest BLACAT1 expression (Fig. 1C; $\mathrm{P}<0.05$ ). Taken together, these findings demonstrated that BLACAT1 may have a prominent role in glioma carcinogenesis.

LncRNA BLACAT1 inhibition decreases glioma cell proliferation. To further explore the roles of BLACAT1 in glioma, both U251 and A172 cells were transfected with si-BLACAT1 or si-NC, and one si-RNA (si-BLACAT1-1) with a high interference efficiency was selected for subsequent experiments (Fig. 2A; $\mathrm{P}<0.05$ ). CCK-8 assay demonstrated that BLACAT1 inhibition significantly suppressed glioma cell proliferation compared with the si-NC group (Fig. 2B; $\mathrm{P}<0.05)$. Colony formation assay similarly indicated that colony numbers of glioma cells transfected with si-BLACAT1 were significantly inhibited compared with the si-NC group (Fig. 2C; $\mathrm{P}<0.05$ ). In addition, flow cytometry analysis demonstrated that BLACAT1 inhibition significantly increased glioma cell apoptosis compared with si-NC group (Fig. 2D; $\mathrm{P}<0.05$ ).

LncRNA BLACATl inhibition decreases glioma cell invasion. Wound-healing assay indicated that BLACAT1 inhibition significantly decreased the migration ability of glioma cells compared with the control (Fig. 3A; $\mathrm{P}<0.05$ ). Transwell invasion assay indicated that BLACAT1 inhibition reduced the invasion ability of glioma cells (Fig. 3B; $\mathrm{P}<0.05$ ). Epithelial-mesenchymal-transition (EMT) is an important process for tumor metastasis therefore in the present study the effects of BLACAT1 on EMT processes were investigated.
Results demonstrated that BLACAT1 inhibition decreased mRNA and protein levels of N-cadherin and vimentin whilst E-cadherin mRNA and protein levels increased (Fig. 3C and $\mathrm{D} ; \mathrm{P}<0.05)$. These findings demonstrated that BLACAT1 knockdown inhibited glioma cell proliferation and invasion in vitro.

LncRNA BLACATl activates Wnt/ $\beta$-catenin signaling in glioma. To further explore the BLACAT1-mediated molecular mechanism, effects of BLACAT1 on the Wnt/ $\beta$-catenin pathway were investigated. Results demonstrated that BLACAT1 knockdown significantly decreased the $\beta$-catenin and cyclin D1 mRNA and protein levels in both U251 and A172 cells (Fig. 4A and B; $\mathrm{P}<0.05$ ). These findings indicated that BLACAT1 may serve as a novel oncogenic lncRNA by regulating the $\mathrm{Wnt} / \beta$-catenin signaling pathway in glioma.

\section{Discussion}

Initiation and development of glioma involves both genetic and epigenetic alterations (16). LncRNAs are a class of non-coding RNAs, which have important roles under both physiological and pathological conditions (7). BLACAT1 is present on human chromosome 1q32.1 and has a transcript of 2,616 kb (17). Recent studies demonstrated that BLACAT1 may have important roles in tumor progression. For example, Shan et al (18) determined that lncRNA BLACAT1 promotes cell proliferation and invasion in human cervical cancer. Ye et al (19) identified that lncRNA BLACAT1 promotes the proliferation, migration, and invasion of non-small cell lung cancer through sponging miR-144. Wu et al (20) revealed that lncRNA BLACAT1 modulates ABCB1 to promote oxaliplatin resistance of gastric cancer via sponging miR-361. However, Liao et al (21) demonstrated that lncRNA BLACAT1 is downregulated in papillary thyroid carcinoma and could act as a suppressor gene in tumor progression. The expression and underlying mechanisms of lncRNA BLACAT1 in glioma progression remains unclear. The present study demonstrated that lncRNA BLACAT1 was highly expressed in glioma tissues and cell lines. In addition, lncRNA BLACAT1 inhibition reduced proliferation, migration, and invasion of glioma cells.

EMT has been proposed as a key process in the induction of tumor cell invasion and metastasis (22). For example, 


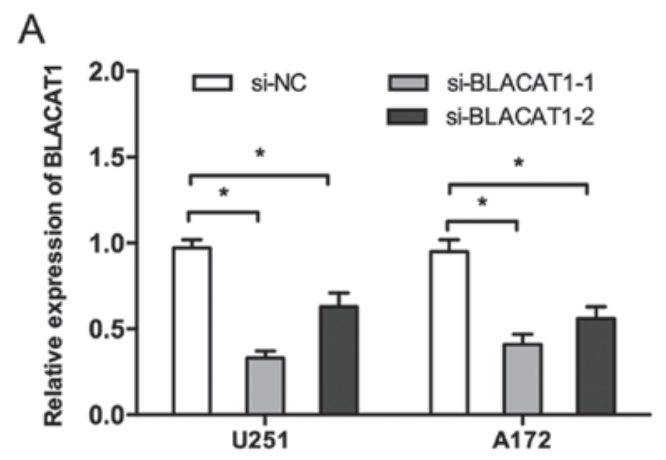

B
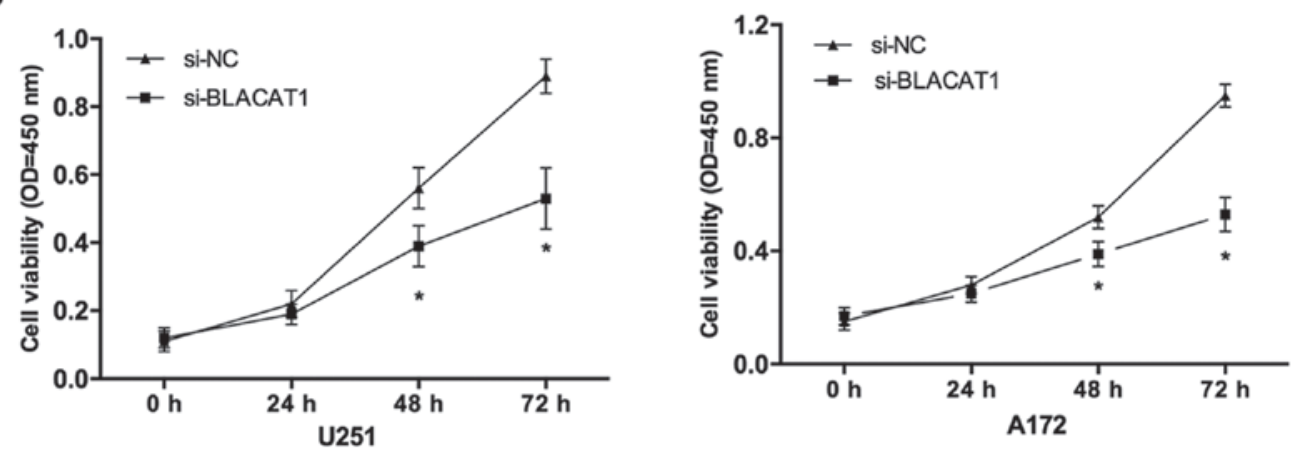

C U251
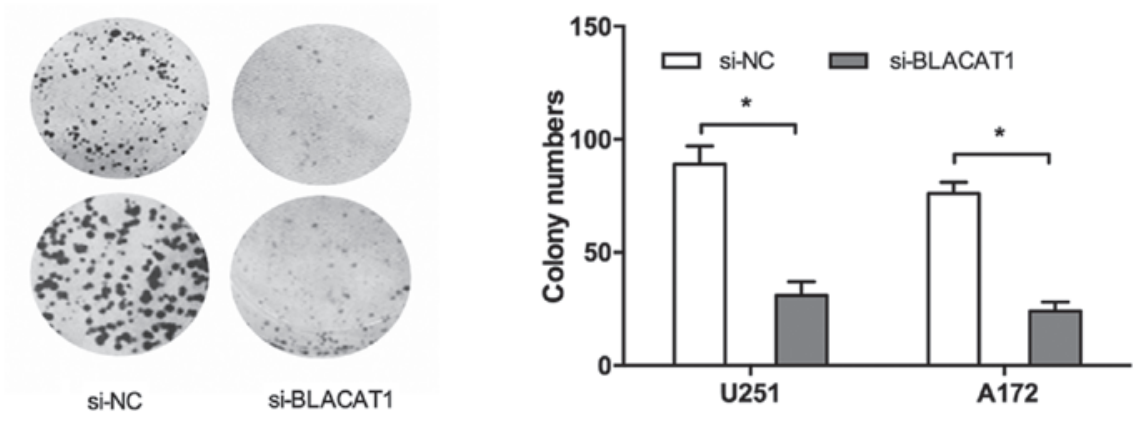

D
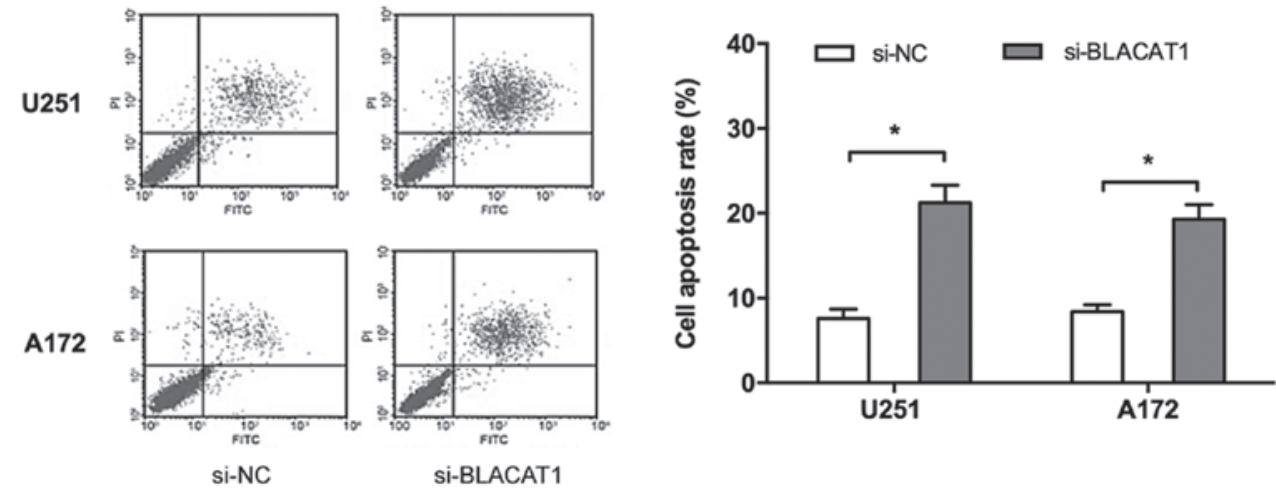

Figure 2. BLACAT1 promotes glioma cell proliferation. (A) Efficacy of BLACAT1 knockdown in U251 and A172 cells by siRNAs as determined by RT-qPCR (B) Cell viability of U251 and A172 cells was determined by CCK-8 assay and (C) colony formation assay. (D) The apoptosis of U251 and A172 cells was determined by flow cytometry. "P<0.05 vs. si-NC. BLACAT1, bladder cancer associated transcript 1; siRNA, small interfering RNA, RT-qPCR, reverse transcription-quantitative polymerase chain reaction; $\mathrm{NC}$, negative control.

Li et al (23) determined that IncRNA SLC25A25 antisense RNA 1 inhibition promotes proliferation, chemoresistance, and EMT in colorectal cancer cells. He et al (24) demonstrated that lncRNA FEZF1 antisense RNA 1 enhances EMT processes through suppressing E-cadherin expression and regulating the WNT pathway in non-small cell lung cancer. The present study demonstrated that BLACAT1 inhibition suppressed EMT processes by decreasing expression of the mesenchymal 
A
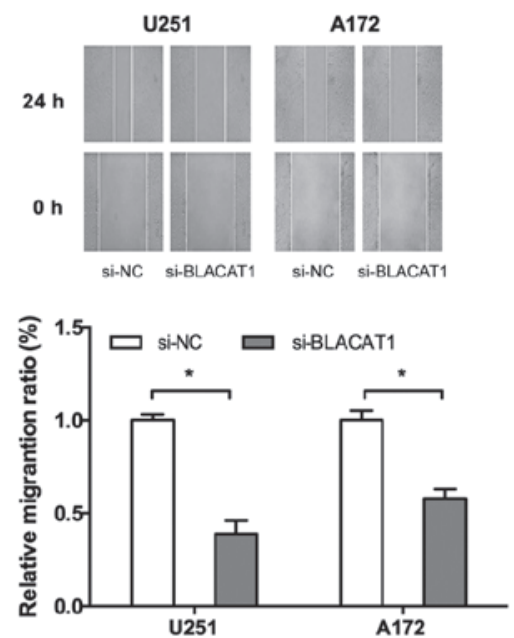

B
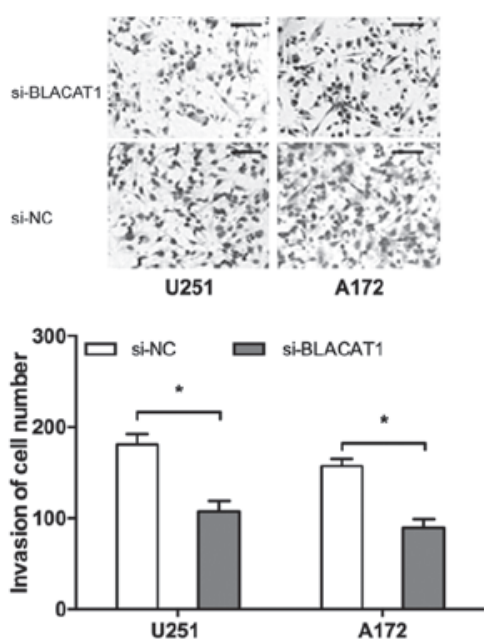

C

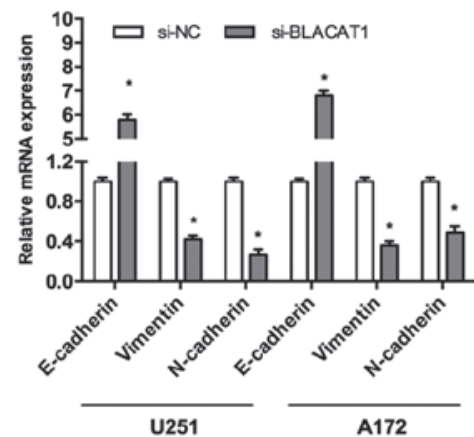

D
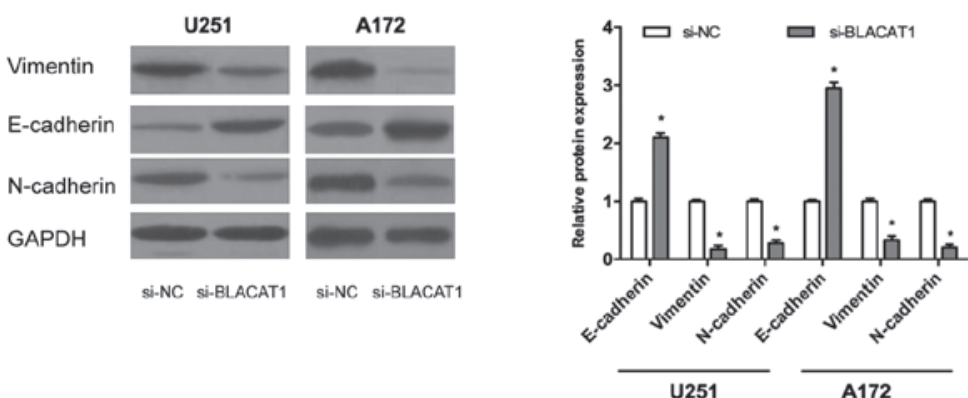

Figure 3. BLACAT1 promotes glioma cell invasion and EMT. (A) The migration ability of U251 and A172 cells was determined by wound-healing assay. (B) The invasion ability of U251 and A172 cells was determined by Transwell invasion assay. (C) Reverse transcription-quantitative polymerase chain reaction and (D) western blot analysis were used to determine N-cadherin, E-cadherin, and vimentin expression in glioma cells. "P $<0.05$ vs. si-NC. BLACAT1, bladder cancer associated transcript 1; EMT, epithelial-mesenchymal-transition; siRNA, small interfering RNA; NC, negative control.

A

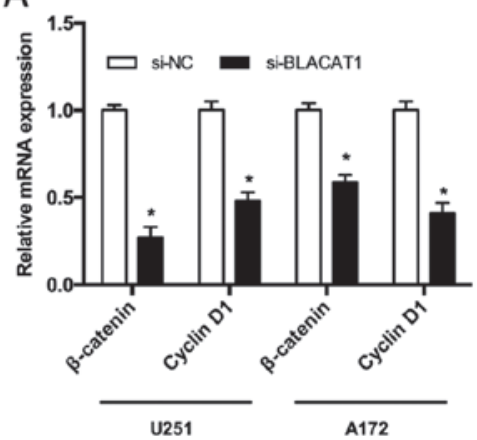

B

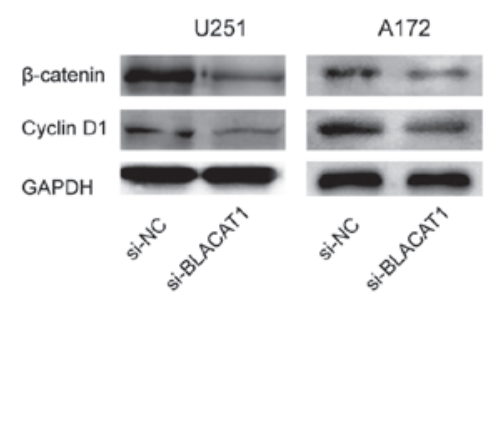

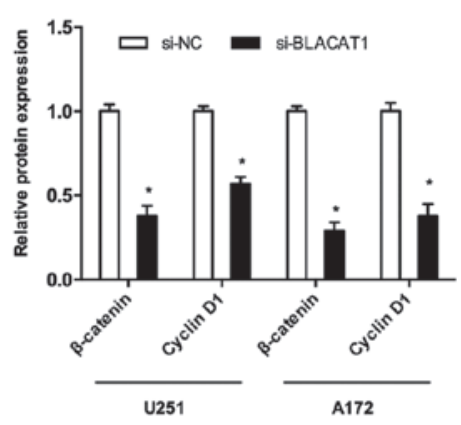

Figure 4. BLACAT1 promotes the Wnt/ $\beta$-catenin pathway in glioma cells. (A) Reverse transcription-quantitative polymerase chain reaction was used to determine $\beta$-catenin and cyclin D1 mRNA expression in glioma cells, following BLACAT1 silencing. (B) Western blot analysis was used to determine $\beta$-catenin and cyclin D1 protein expression in glioma cells. "P $<0.05$ vs. si-NC. BLACAT1, bladder cancer associated transcript 1 ; siRNA, small interfering RNA; NC, negative control.

markers $\mathrm{N}$-cadherin and vimentin whilst increasing expression of the epithelial marker E-cadherin. The mechanism involved in the regulative effects of BLACAT1 on EMT processes was then investigated.

The Wnt/ $\beta$-catenin pathway has been reported to have an important role in EMT (25). For example, Tian et al (26) demonstrated that miR-361-5p inhibits the mobility of gastric cancer cells through suppressing EMT via the Wnt/ $\beta$-catenin pathway. Yuan et al (27) identified that rhomboid 5 homolog 1 regulates the APC-mediated stimulation of EMT and also proliferation of colorectal cancer cells via the Wnt/ $\beta$-catenin pathway. The present study investigated whether BLACAT1 modulated the Wnt/ $\beta$-catenin pathway in glioma cells. Results demonstrated that BLACAT1 inhibition reduced $\beta$-catenin and cyclin D1 mRNA and protein expression levels. Based on these findings, BLACAT1 might promote EMT processes, at least partly, via activating the $\mathrm{Wnt} / \beta$-catenin pathway in glioma. 
In conclusion, these results determined that lncRNA BLACAT1 was upregulated in glioma, and highly expressed BLACAT1 promoted cellular proliferation and invasion. These effects may be related to increased EMT-related gene expression via promotion of the $\mathrm{Wnt} / \beta$-catenin pathway. The present findings revealed that BLACAT1 might be a novel therapeutic target for glioma treatment.

\section{Acknowledgements}

Not applicable.

\section{Funding}

No funding was received.

\section{Availability of data and materials}

All data generated or analyzed during this study are included in this published article.

\section{Authors' contributions}

$\mathrm{XL}$ and JW conceived and designed the study, and analyzed and interpreted the results. XL, SQ, DM and JF performed experiments and wrote this manuscript. All authors read and approved the final manuscript.

\section{Ethics approval and consent to participate}

For the use of human samples, the protocol for the present study was approved by the Institutional Ethics Committee of Affiliated Hospital of Hebei University of Engineering, and all enrolled patients signed a written informed consent document.

\section{Patient consent for publication}

All patients within the present study provided consent for the publication of their data.

\section{Competing interests}

The authors declare that they have no competing interests.

\section{References}

1. Siegel RL, Miller KD and Jemal A: Cancer statistics, 2016. CA Cancer J Clin 66: 7-30, 2016.

2. Schwartzbaum JA, Fisher JL, Aldape KD and Wrensch M: Epidemiology and molecular pathology of glioma. Nat Clin Pract Neurol 2: 494-503, 2006.

3. Trabelsi S, Brahim DH, Ladib M, Mama N, Harrabi I, Tlili K, Yacoubi MT, Krifa H, Hmissa S, Saad A and Mokni M: Glioma epidemiology in the central tunisian population: 1993-2012. Asian Pac J Cancer Prev 15: 8753-8757, 2014.

4. Wrensch M, Fisher JL, Schwartzbaum JA, Bondy M, Berger M and Aldape KD: The molecular epidemiology of gliomas in adults. Neurosurg Focus 19: E5, 2005.

5. Reich DE, Cargill M, Bolk S, Ireland J, Sabeti PC, Richter DJ, Lavery T, Kouyoumjian R, Farhadian SF, Ward R and Lander ES: Linkage disequilibrium in the human genome. Nature 411: 199-204, 2001.

6. Lander ES: Initial impact of the sequencing of the human genome. Nature 470: 187-197, 2011.
7. Mercer TR, Dinger ME and Mattick JS: Long non-coding RNAs: Insights into functions. Nat Rev Genet 10: 155-159, 2009.

8. Shi X, Sun M, Liu H, Yao Y and Song Y: Long non-coding RNAs: A new frontier in the study of human diseases. Cancer Lett 339: 159-166, 2013.

9. Monnier P, Martinet C, Pontis J, Stancheva I, Ait-Si-Ali S and Dandolo L: H19 lncRNA controls gene expression of the Imprinted Gene Network by recruiting MBD1. Proc Natl Acad Sci U S A 110: 20693-20698, 2013.

10. Lee JT: Epigenetic regulation by long noncoding RNAs. Science 338: 1435-1439, 2012.

11. Zhou M, Wang X, Shi H, Cheng L, Wang Z, Zhao H, Yang L and Sun J: Characterization of long non-coding RNA-associated ceRNA network to reveal potential prognostic lncRNA biomarkers in human ovarian cancer. Oncotarget 7: 12598-12611, 2016.

12. Feng W, Li L, Xu X, Jiao Y and Du W: Up-regulation of the long non-coding RNA RMRP contributes to glioma progression and promotes glioma cell proliferation and invasion. Arch Med Sci 13: 1315-1321, 2017.

13. Wang XP, Shan C, Deng XL, Li LY and Ma W: Long non-coding RNA PAR5 inhibits the proliferation and progression of glioma through interaction with EZH2. Oncol Rep 38: 3177-3186, 2017.

14. Ma Y, Zhou G, Li M, Hu D, Zhang L, Liu P and Lin K: Long noncoding RNA DANCR mediates cisplatin resistance in glioma cells via activating AXL/PI3K/Akt/NF- $\kappa \mathrm{B}$ signaling pathway. Neurochem Int 118: 233-241, 2018.

15. Livak KJ and Schmittgen TD: Analysis of relative gene expression data using real-time quantitative PCR and the 2(-Delta Delta C(T)) method. Methods 25: 402-408, 2001.

16. Alonso MM, Diez-Valle R, Manterola L, Rubio A, Liu D, Cortes-Santiago N, Urquiza L, Jauregi P, Lopez de Munain A, Sampron N, et al: Genetic and epigenetic modifications of Sox2 contribute to the invasive phenotype of malignant gliomas. PLoS One 6: e26740, 2011.

17. Chen X, Dai M, Zhu H, Li J, Huang Z, Liu X, Huang Y, Chen J and Dai S: Evaluation on the diagnostic and prognostic values of long non-coding RNA BLACAT1 in common types of human cancer. Mol Cancer 16: 160, 2017.

18. Shan D, Shang Y and Hu T: Long noncoding RNA BLACAT1 promotes cell proliferation and invasion in human cervical cancer. Oncol Lett 15: 3490-3495, 2018.

19. Ye JR, Liu L and Zheng F: Long noncoding RNA bladder cancer associated transcript 1 promotes the proliferation, migration, and invasion of nonsmall cell lung cancer through sponging miR-144. DNA Cell Biol 36: 845-852, 2017.

20. Wu X, Zheng Y, Han B and Dong X: Long noncoding RNA BLACAT1 modulates ABCB1 to promote oxaliplatin resistance of gastric cancer via sponging miR-361. Biomed Pharmacother 99: 832-838, 2018.

21. Liao D, Lv G, Wang T, Min J, Wang Y and Liu S: Prognostic value of long non-coding RNA BLACAT1 in patients with papillary thyroid carcinoma. Cancer Cell Int 18: 47, 2018.

22. Yang $\mathbf{J}$ and Weinberg RA: Epithelial-mesenchymal transition: At the crossroads of development and tumor metastasis. Dev Cell 14: 818-829, 2008.

23. Li Y, Huang S, Li Y, Zhang W, He K, Zhao M, Lin H, Li D, Zhang $\mathrm{H}$, Zheng $\mathrm{Z}$ and Huang $\mathrm{C}$ : Decreased expression of LncRNA SLC25A25-AS1 promotes proliferation, chemoresistance, and EMT in colorectal cancer cells. Tumor Biol 37: 14205-14215, 2016.

24. He R, Zhang FH and Shen N: LncRNA FEZF1-AS1 enhances epithelial-mesenchymal transition (EMT) through suppressing E-cadherin and regulating WNT pathway in non-small cell lung cancer (NSCLC). Biomed Pharmacother 95: 331-338, 2017.

25. Nusse R and Clevers $H$ : Wnt/ $\beta$-catenin signaling, disease, and emerging therapeutic modalities. Cell 169: 985-999, 2017.

26. Tian L, Zhao Z, Xie L and Zhu J: MiR-361-5p inhibits the mobility of gastric cancer cells through suppressing epithelial-mesenchymal transition via the Wnt/ $\beta$-catenin pathway. Gene 675: 102-109, 2018.

27. Yuan H, Wei R, Xiao Y, Song Y, Wang J, Yu H, Fang T, Xu W and Mao S: RHBDF1 regulates APC-mediated stimulation of the epithelial-to-mesenchymal transition and proliferation of colorectal cancer cells in part via the Wnt/ $\beta$-catenin signalling pathway. Exp Cell Res 368: 24-36, 2018. 